\title{
Vibration Analysis for Anomaly Detection in Unmanned Aircraft
}

\author{
O. Bektash and Anders la Cour-Harbo \\ Department of Electronic Systems, Aalborg University, Denmark \\ o.bektash@es.aau.dk \\ alc@es.aau.dk
}

\begin{abstract}
Unmanned aircraft systems are increasingly operating in sensitive airspace which involves risks about the implications of drone activity. As a result, managing safer operations will be crucial to success for the operators. Vibration analysis can provide a detailed examination of drone health status by examining signal levels and frequencies. Thus, this paper investigates how vibration measurements from drone flights can be analysed to infer the condition of the mechanical integrity of the drone. The method should ascertain whether a vibration analysis algorithm can detect major fault progress in drone flights. In order to track and monitor the anomalies on the drone, the research proposes the periodogram method on the vibration data from on-board vibration sensors. The method was tested with an unmanned aircraft with and without full payload, and the results provide support for the proposed algorithm, with the ability to determine anomaly from an unsteady flight but those results being preliminary to further research. This suggests that further drone safety research can use the same signal processing method regarding vibration related anomalies.
\end{abstract}

Keywords: Vibration analysis, fault detection, in-flight monitoring, signal processing, automated response, Drone safety

\section{INTRODUCTION}

Drones are subjected to various extents of vulnerabilities during operation, including failures constituting risks for individuals, inhabited areas, places with high security concerns and societal acceptance. These push the regulations to enforce safe, sustainable, and secure operations during which it is intended to provide a less restricted drone use (EU, 2019a,b). Widely considered to be a good way to deal with the operational failures is estimating whether any fault has occurred or about to occur. Such estimations have been widely adopted in the fields of "Predictive Maintenance" and "Prognostics and

O. Bektash et al. This is an open-access article distributed under the terms of the Creative Commons Attribution 3.0 United States License, which permits unrestricted use, distribution, and reproduction in any medium, provided the original author and source are credited.
Health Management (PHM)". These fields are maturing with a wealth of progressing methods and algorithms that inspect, cleanse and transform the monitored signals such as temperature limits, current, pressure, or even a setting to any physical quantity which might be considered critical for the system safety (Goebel et al., 2019).

\subsection{Literature review}

One of the major topics investigated in PHM of unmanned aircraft system (UAS) is prognostics of the remaining flying time at which the UAS can no longer complete the expected operational tasks. Using prognostics generally involve estimation of the time ahead to critical outcomes such as remaining time to empty drone battery, or a set of features from monitored signals which constitute little or no explicit degradation signification, but somehow correlated with the conditions that might possess a risk to the drone safety.

PHM in UAS has gradually broadened and closely follows various paradigms such as safety modelling. For example, Saha et al. (2011) provided evidence for a novel health management system based on a Bayesian inference driven prognostic model which can provide end-of-discharge estimation for electric UAS. In light of the reported absence of statistically significant flight data, it is reasonable to assume that the work developed a discharge model for batteries rather than motivating data-driven approaches. As a matter of fact, there are numerous UAS prognostic frameworks which also conceptually approach the safety challenges and look out on to non-data driven frameworks. In a later work, Saha et al. (2012) presented a stochastic programming scheme using a model parameter augmented Particle Filtering framework to analyse UAS battery performance under some load uncertainties. Comparatively, Balaban \& Alonso (2013) carried out partially observable Markov decision processes and its application to UAS mission planning for prognostic decision making with complex operating conditions and non-linear degradation uncertainties. A more comprehensive validation by data can be found in the work of Goebel \& Saha (2013) which presents prognostics for component degradation estimation and performs an implementation on an electric UAS propul- 
sion. The work employed a model-based Particle Filtering methodology which was linked to the internal processes of the battery but also validated by experimental data. By emphasising the need for developing data-driven models, Eleftheroglou et al. (2019) also utilised data for challenges of Lithium-Polymer (Li-Po) batteries for UAS. Their work addressed diagnostic and prognostic measures by using several discharge voltage information on a data-driven approach of a Hidden Semi Markov model variant.

The health management of drones needs to be assessed to a broader extent because there is always a potential risk of failing to understand the drone failures. That being the case, one should consider the operational features derived from condition monitoring data that can provide health indicators to detect and diagnose the degradation in the drone performance. A well-known example of such features may be calculated from a vibration power spectrum as it can provide advanced analytics from measured observations (Goebel et al., 2019). A study by Bechoefer \& Capt (2004) allowed predicting impending failures of rotorcraft drivetrains by analysing vibration and acoustic emissions data, resulting in the progress toward relatively advanced Health and Usage Monitoring Systems (Goebel \& Saha, 2013).

For unmanned aircraft, earlier research showed an application of vibration data analysis techniques in an early model UAS (Bennett \& Abel, 1982). The results were presented in a flight flutter test of a drone wing. In more recent work of Simsiriwong \& Sullivan (2012), further contributions were made in vibration analysis of a composite UAS wing. These studies on drone vibrations showed various explorations in multiple domains, some focusing on the effects of vibration in mounted systems, others on the body vibration in drones. Lai et al. (2012) brought some information about the analysis of noncommutativity errors of strapdown inertial navigation system under the vibration in UAS. Vibration environment assessment of UAS has a potential for further development and hence supported by further studies.

For example, Plasencia et al. (2012) deployed a vibration analysis in an unmanned helicopter to study the effects of vibrations on an on-board vision system. Similarly, other works have developed the use of body vibration to analyse the drone. Verbeke \& Debruyne (2016) proposed a work of numerical vibration analysis into a small UAS multirotor frame. Their framework allowed critical decisions such as finding low-vibration locations in which sensitive electronics can be mounted.

Nguyen et al. (2017) extended the use of body vibration to the detection of drone presence in critical airspace where the unmanned operations may lead to harm. They examined the body vibration as a unique physical characteristics of the drone for a cost-effective detection system. In a categorically different choice of vibration analysis, Herrmann et al. (2019) proposed a structural vibration analysis as exemplified on a drone based mobile sensing platform. However, this work was based on the identification of the dynamic structure behaviours such as a wind turbine tower. That is to say, they use drones to study structures rather than analysing the drone vibrations.

There is an incomplete picture of how to develop a vibrationbased prognostics and predictive maintenance methodology for drone operations. Actually, vibration analysis has been regarded as a common condition monitoring scheme used in the industry for machinery systems and an effective tool for fault diagnosis and prognosis (Amirat et al., 2009; Yang et al., 2009; Chen et al., 2015; Saidi et al., 2017). Even though the vibration based methods in the literature are practised to detect rotating equipment faults with various techniques (Yang et al., 2009; Ali et al., 2015; Saidi et al., 2017; Ali et al., 2018), they also carry the potential of identifying the safety risks of flying drones. They can estimate the health indicator and make the detection and diagnosis of drone performance degradation for decision making. Therefore, in order to estimate operational drone failures successfully and develop more sophisticated procedures for in-flight anomaly detection, this study aims a comprehensive understanding of vibration signatures associated with flight anomalies.

\subsection{Current work}

Granting the fact that an extensive literature has developed on vibration based data driven diagnostics and prognostics, this paper responds to the call for an original approach about overall response system which simultaneously monitors the vibration data and determines the anomalies and health status of the drone. This is visualised in Figure 1 where the steps are shown chronologically. This representation follows a standard predictive maintenance workflow: start of the operations with initial conditions and a performance level, signal monitoring and exploration, feature extraction from the raw data, state estimation, and performance analysis.

First, the framework involves the process of recording drone vibrations with the aim of detecting a failure which may be an indicator of in-flight anomalies or even a developing system failure. Then, the monitored vibration signals are engaged to calculate various features in the time. In the following phase, a periodogram method for the determination of power spectra is performed by segmenting the continuous vibration data into successive blocks, estimating a periodogram for each of these blocks, and averaging. This is followed by taking the maximum value from each block in an attempt to form a severity indication of the in-flight anomaly or the fault. This allows to detect the significant trends in health change over time and to better understand the signal measurements available during the flight. Finally, the framework can provide information for further decision making. 


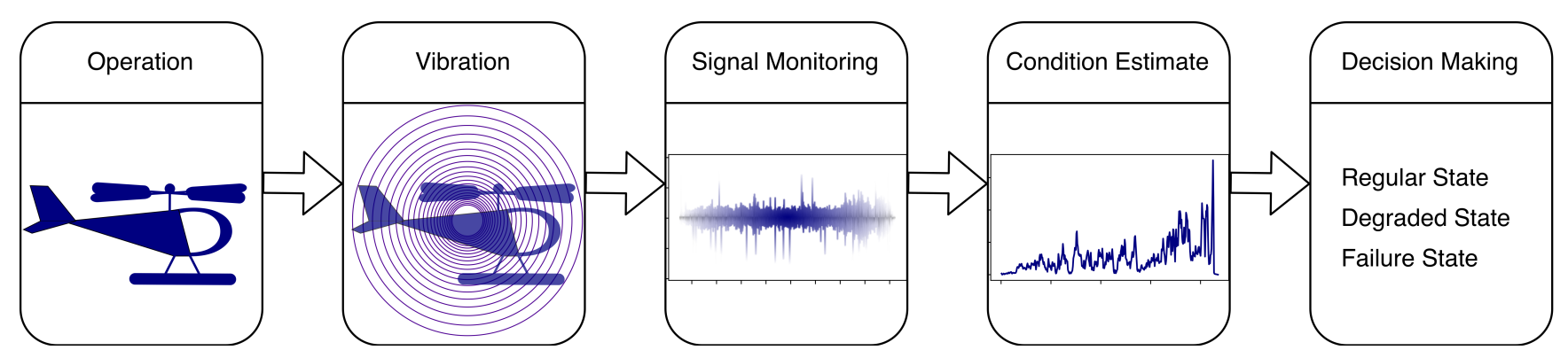

Figure 1. Main steps in vibration signal processing for in-flight monitoring.

The rest of the paper is organised as follows: First, the description of the drone and test flight program is presented. This is followed by a presentation of the methodology with procedures used in the framework. The results and findings of the study are then summarised. Finally, implications, limitations, and directions for future studies are laid out in the conclusion section.

\section{Drone and Test flight Program}

The data set was recorded on September 17, 2019, at a small airfield in Denmark. The data come from a series of sensors mounted on a T50 rotorcraft from Aveox. This is a 90 $\mathrm{kg}$ maximum take-off weight turbine-powered rotorcraft with dual rotors (Flettner system). A total of four flights were conducted over a period of about 2 hours, each flight lasting about 10 minutes. All flights were mostly in hover. The purpose was to determine the level of vibration of the aircraft in different flight conditions (with no payload and with full payload, as well as in strong wind). It was fairly windy that day, and all flights were conducted in wind speed between 5 and $20 \mathrm{~m} / \mathrm{s}$ (varying over the flight period and with altitude).

From previous flights, it was observed that when fully loaded the aircraft exhibited severe vibrations beyond what is acceptable, and the purpose of the second flight was to determine more accurately how severe the problem was. After the flights were conducted and after consultation with the manufacturer, the problem has largely been remedied by a change to the rotor blade and rotor hub configuration. As such, the vibration data from the flight are indeed typical for a minor malfunction that could have developed into a more serious issue of structural degradation and ultimate disintegration.

The four flights were conducted from the same take-off position and to an altitude around 25 meters, with the last flight being higher to increase the wind speed exerted on the aircraft. Changes were made to the aircraft between flights as follows:

1. Initial flight with no payload to establish a baseline. Altitude around $25 \mathrm{~m}$.

2. $35 \mathrm{~kg}$ payload (steel plates) were fixed to the bottom of the fuselage. Altitude around $25 \mathrm{~m}$.
3. The $35 \mathrm{~kg}$ payload was removed, and a hook was installed, and a $5 \mathrm{~kg}$ payload in a 4 meter long metal chain was attached. Altitude around $25 \mathrm{~m}$.

4. The $5 \mathrm{~kg}$ payload was unhooked, and no other payload installed. This flight was hover at $25 \mathrm{~m}, 50 \mathrm{~m}$, and $75 \mathrm{~m}$, with the nose as well as the right side into the wind.

To record the vibrations, a series of identical IMU sensors (SparkFun 9DoF Razor IMU) were located throughout the aircraft.

All seven sensors were fixed to the aircraft in locations as shown in Figure 2 in random orientations, since only the magnitude is of interest to us. Each sensor records translational acceleration, angular velocity, and the magnetic field at a rate of $100 \mathrm{~Hz}$. In addition, horizontal wind speed and direction were measured on the aircraft using an FT Technology anemometer at $10 \mathrm{~Hz}$. Although this sensor is located below the aircraft and within the rotor wake, the case can be made (which is beyond the scope of this paper) that the measurements are quite accurate when the wind speed is above approximately $5 \mathrm{~m} / \mathrm{s}$.

The continuous change of wind speed and direction obviously affects the aircraft, as can be seen on the flight videos (UASability, n.d.), and it may also change the vibration pattern due to aerodynamic effects on the rotor. However, the frequency of the wind change is so low that it does not have any noticeable effect on the accelerometer data used for analysing vibrations.

Videos of the test flights are available on the UAS-ability YouTube channel (UAS-ability, n.d.) (which spotlights the project that funded the aircraft), or alternatively, can be searched for "UAS-ability" on YouTube. The videos all start with the date, i.e. "19.09.17", and "FL00x" refers to the flight number as listed above. There are four camera views available; a manual tracking camera, an onboard camera, an overview camera, and a ground station camera (called MGCS). For the third flight, there is also a video of the aircraft taken by another drone. 


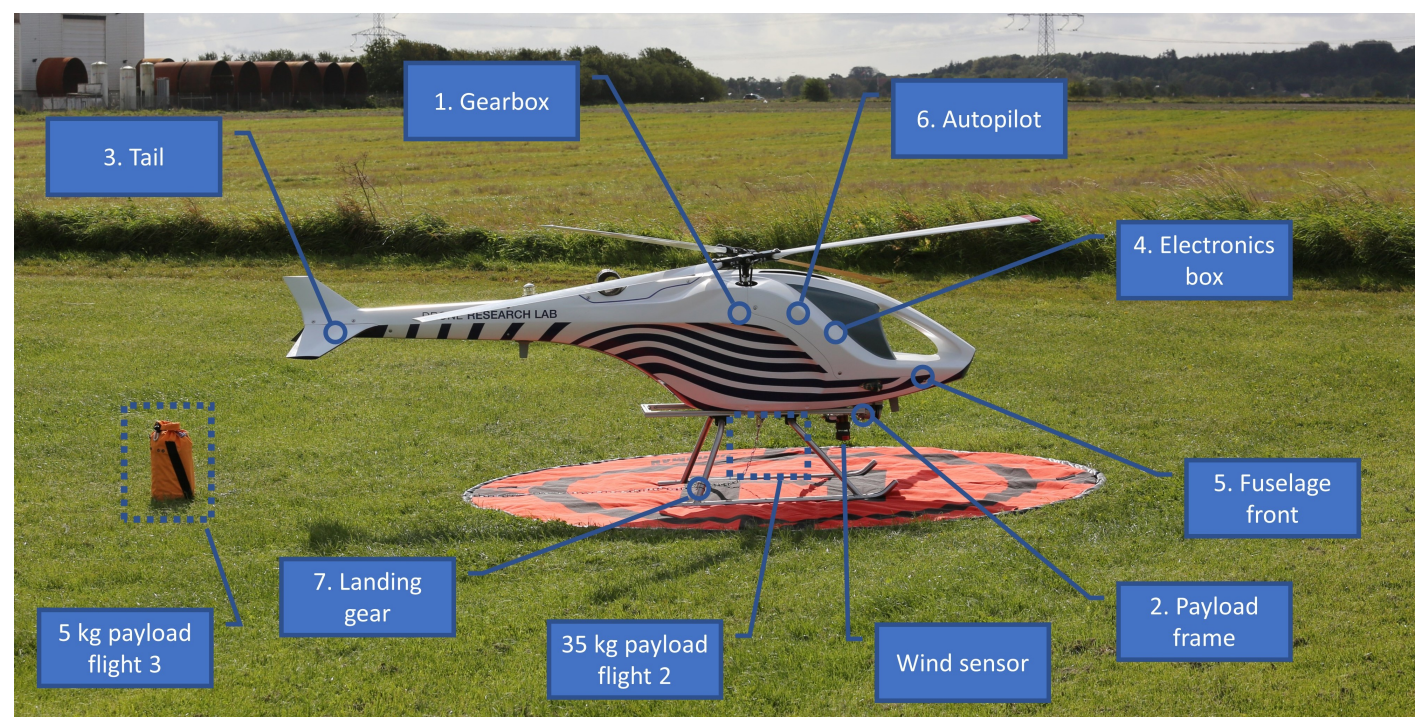

Figure 2. The photo shows the T50 aircraft with the location of the seven IMU sensors, the wind sensors, and the payloads for flight 2 and 3 . The payload for flight 2 is no mounted, but the location is shown.

\section{Methodology}

This section comprises the theoretical analysis of the Welch method for the application of the Fourier transform to the power spectra estimation. The method is used in a sliding window to form a condition indicator of drone flight performance.

A well-known application in digital signal processing is the power spectral estimation of a given data (Barbé et al., 2009). A power spectral density (or simply power spectrum), $P_{x x}(f)$, can be defined as the measure of a signal's power content falling within given frequency bins. A periodogram is based on the definition of such a power spectrum of a signal which can be considered as a finite time series as:

$$
x_{0}, \ldots, x_{N-1}
$$

and the periodogram can be defined by the method of Schuster (1898), formulated as (Proakis, 2001):

$$
P_{x x}(f)=\frac{1}{N}|X(f)|^{2}
$$

Here, $X(f)$ represents the Fourier transform of a sample sequence and it is given by:

$$
X(f)=\sum_{n=0}^{N-1} x(n) e^{-\frac{i 2 \pi}{N} f n}, \quad f=0, \ldots, N-1
$$

According to this, the time series can be decomposed into a spectrum of frequencies.

$$
P_{x x}(f)=\frac{1}{N}\left|\sum_{n=0}^{N-1} x(n) e^{-\frac{i 2 \pi}{N} f n}\right|^{2}
$$

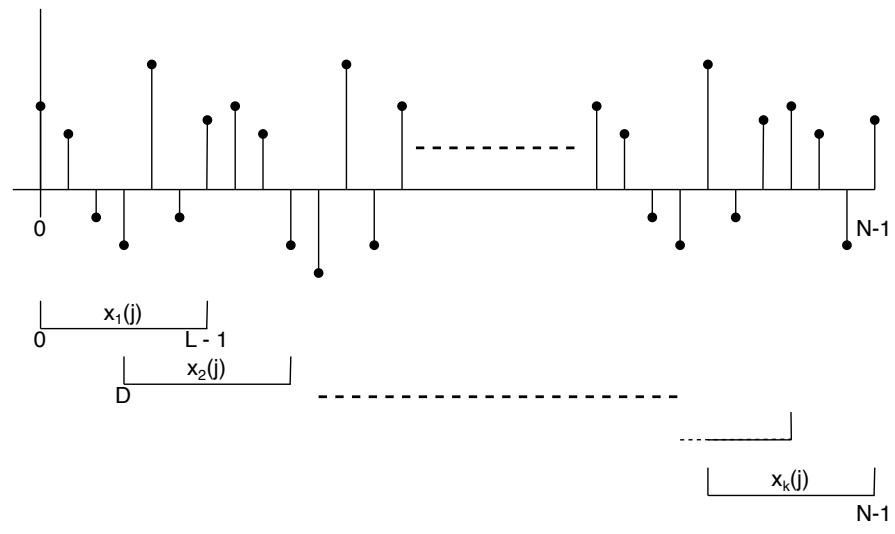

Figure 3. Record segmentation

Welch's method is a modification of this classical periodogram approach (Welch, 1967; Barbé et al., 2009) and it is also often called the periodogram method. It is carried out by dividing the time series into sequential segments, using the periodogram method for each segment, and finding the average of them (Smith III, 2011). Assuming the length of the segments be $L$ and the starting points of them be $D$ steps apart (Welch, 1967), the first segment can be given by:

$$
x_{1}(j)=x(j), \quad j \in\{0,1, \ldots, L-1\}
$$

similarly, the second segment is:

$$
x_{2}(j)=x(j+D), \quad j \in\{0,1, \ldots, L-1\}
$$

Therefore, the total number of observed data, $N$, is:

$$
N=L+D(K-1)
$$


where $\mathrm{K}$ is the total number of segments. The $k$-th segment is defined by

$$
\begin{aligned}
x_{k}(j)=x(j+D(k-1)), & j=0,1, \ldots, L-1 \\
k & =1, \ldots, K
\end{aligned}
$$

Such segmentation of time series is shown in Figure 3.

The second modification by Welch (1967) is to weight the segments by a window

$$
w(j), \quad j=0,1, \ldots, L-1
$$

The segments before the computation of the periodogram form the sequences of:

$$
\begin{aligned}
x_{k}(j) w(j), \quad j & =0,1, \ldots, L-1 \\
k & =1, \ldots, K
\end{aligned}
$$

When the sequences are applied into the periodogram method, the result becomes a "modified" periodogram, $\tilde{P}_{x x}(f)$.

$$
\tilde{P}_{x x}^{(k)}(f)=\frac{1}{L U}\left|\sum_{j=0}^{L-1} x_{k}(j) w(j) e^{-\frac{i 2 \pi}{N} f n}\right|^{2}
$$

where $U$ represents a normalization factor for the power in the window function (Proakis, 2001) and is determined as

$$
U=\frac{1}{L} \sum_{j=0}^{L-1} w^{2}(j)
$$

Finally, the Welch estimate, $P_{x x}^{W}(f)$, takes the mean of these modified periodograms, $\tilde{P}_{x x}(f)$.

$$
P_{x x}^{W}(f)=\frac{1}{K} \sum_{k=1}^{K} \tilde{P}_{x x}^{(k)}(f)
$$

\section{Testing AND Results}

Data are captured on each sensor during flight, copied from there to a common repository and timestamped with an absolute time for comparison. Raw data are recorded from the sensors and contain instrument errors. Once captured, these primary data need to be processed to make the analysis easier during later processing. Every so often, data might include some extreme measures that are beyond the expected range and unlike the other measurements. When modelling, it is needed to clean these outliers to ensure that the observations best illustrate the case. That being the case, the outliers having potential to cause analysis problems are excluded from the primary data set.
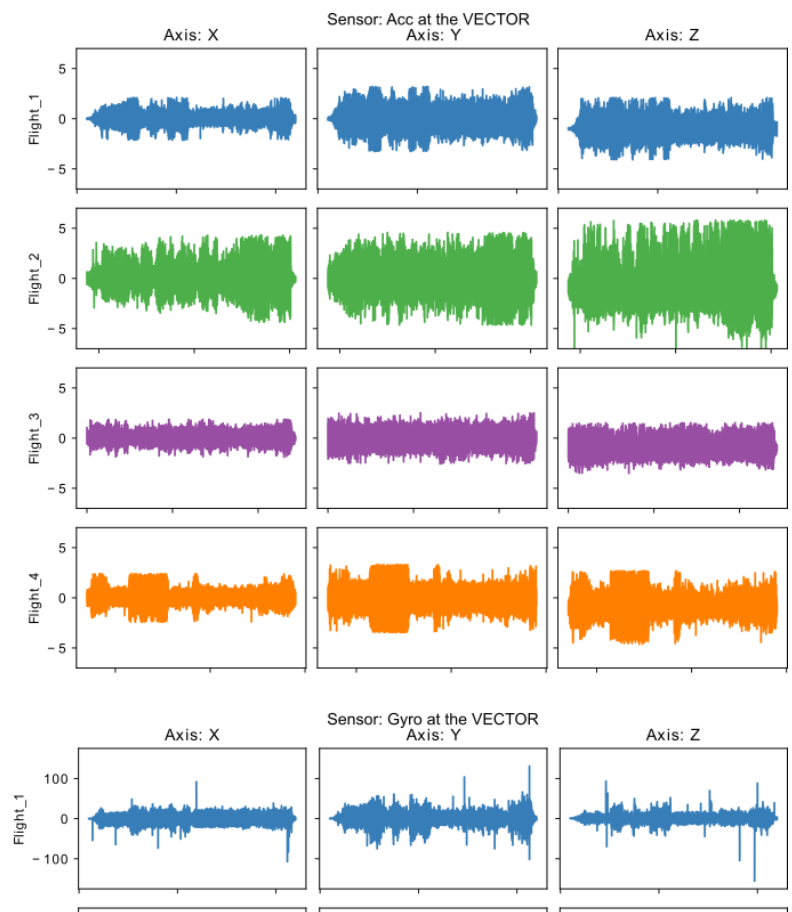

Sensor: Gyro at the VECTOR
Axis: Y $Y$
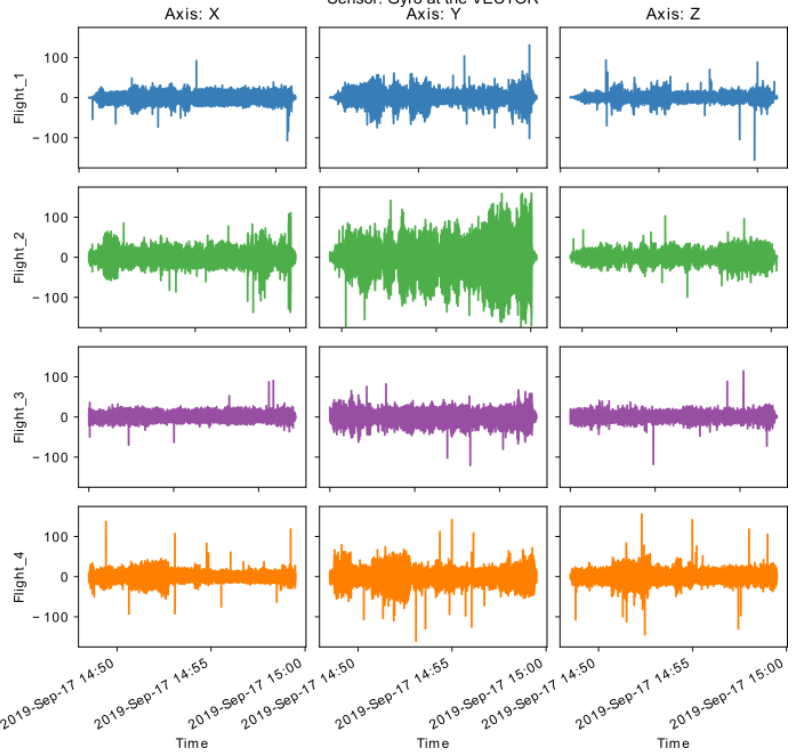

Figure 4. Raw vibration measurements after the outliers removed

Data points that differ significantly from the distribution indicate operational or sensor related error. To find them, the data, $x$, is scaled by measuring the deviation of the observation from the mean of the group. This statistical measurement, called z-score, represents a score's relationship to the mean in a group of scores and represented as:

$$
z=\frac{x-\mu}{\sigma}
$$

When the value of a scaled data point or observation is significantly higher than the mean (such as $\left|z_{i}\right|>3.5$ ), it is replaced by using linear interpolation between two neighbour points.

Figure 4 provides a representation of raw data after the outlier removal. Of the 7 IMUs mounted on the aircraft, the one 


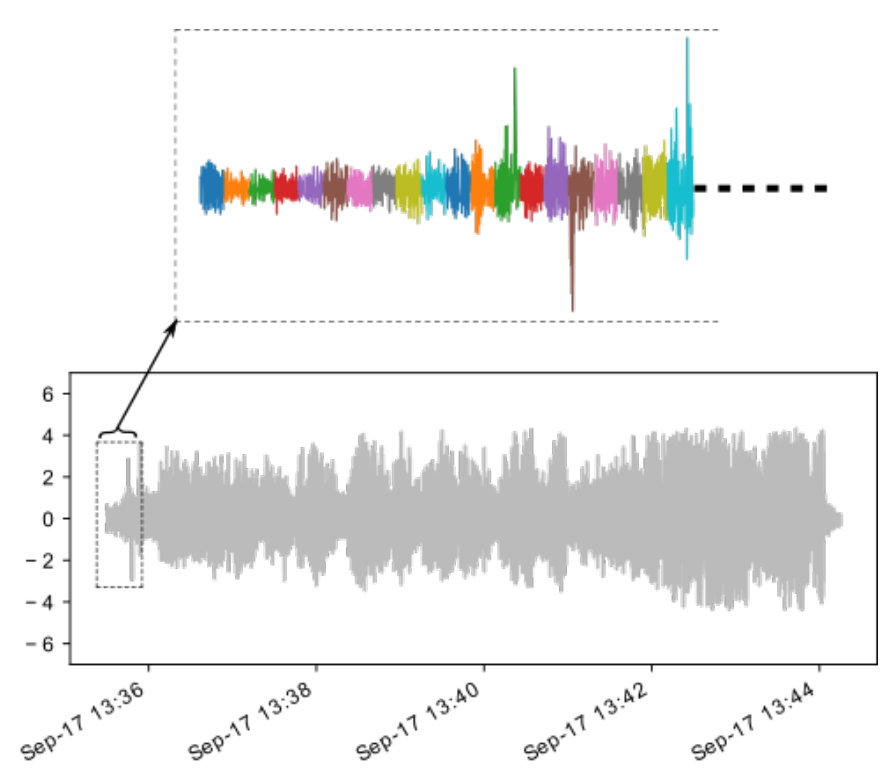

Figure 5. Data segmentation into cycle to be applied into Welch method

most representative for what would be available on a normal unmanned aircraft, is the IMU mounted directly on the autopilot (which is called VECTOR in this aircraft). This is because it resembles what the autopilot mounted on dampeners would typically measure. Therefore, the data from this IMU is used in the following.

To apply Welch's method, the data is segmented by dividing the recorded time series up and grouping sequentially into cycles so that the framework can use it more efficiently. These cycles are represented by different colours in Figure 5 and the proposed periodogram method will be applied into them individually.

Figure 6 gives an indication of how the power spectral density for every single segmented cycle varies with frequency. Each power spectrum is estimated by dividing the cycles into overlapping segments, calculating a modified periodogram for each segment and averaging them. Even though anomaly severity might be indicated in the sequential colouring, (from green to yellow), it is not certain whether this is caused by an increasing fault as the anomaly was due to the high payload. From the short review from this Figure, it is observed that the power spectrum values are significantly higher during the second flight compared to the other three flights. Statistical features of these periodograms can be potential indicators of the flight anomaly.

When they are extracted, the obtained features will be associated with the overall system performance degradation. However, the statistical features may include unwanted and unrelated noise that the reformatted series may suffer during capture. Such noise might carry misleading and non-useful information, even if they are not interfering with other mea-
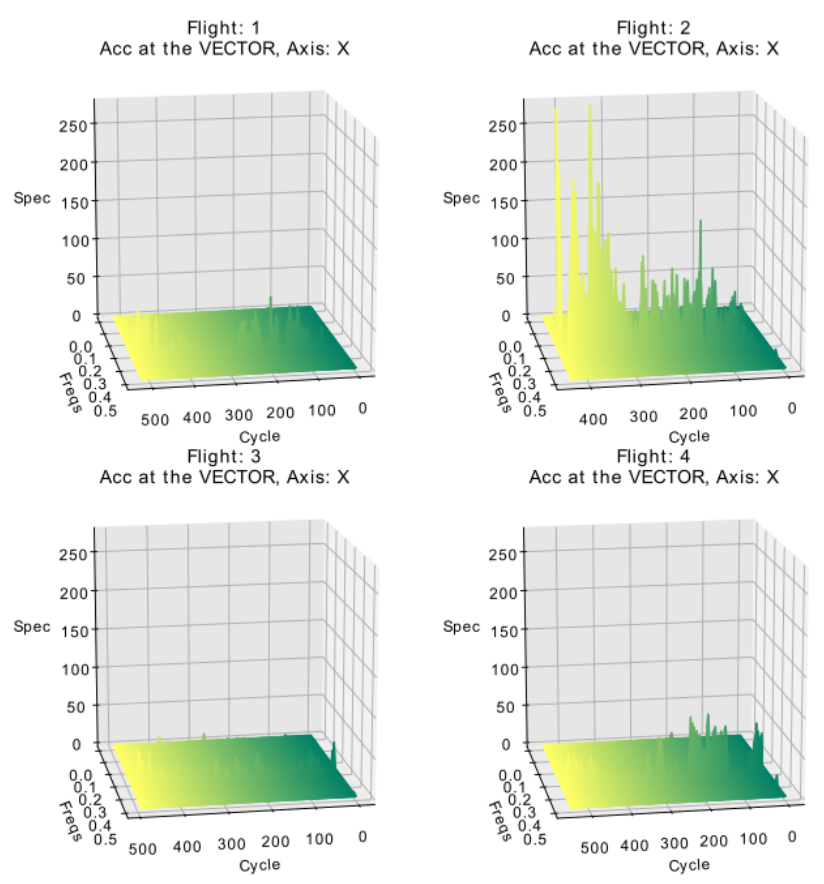

Figure 6. Estimations of Welch's Method from the segmented cycle data

surements. To highlight longer-term trends and smooth out short-term fluctuations, a rolling mean filter with a lag window of 5 steps is applied to the statistical features extracted from the periodograms.

Table 1. Mean and standard deviation of $\max (\mathrm{spec})$

Flight $1 \quad$ Flight 2 Flight $3 \quad$ Flight 4

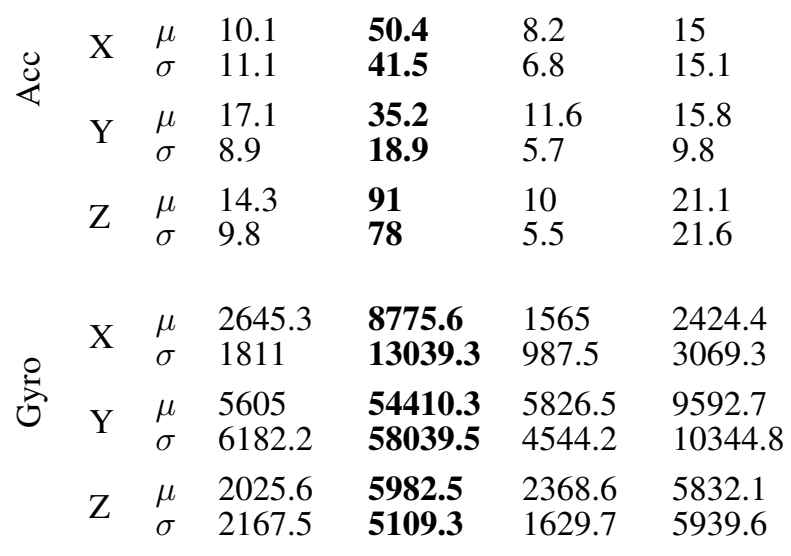



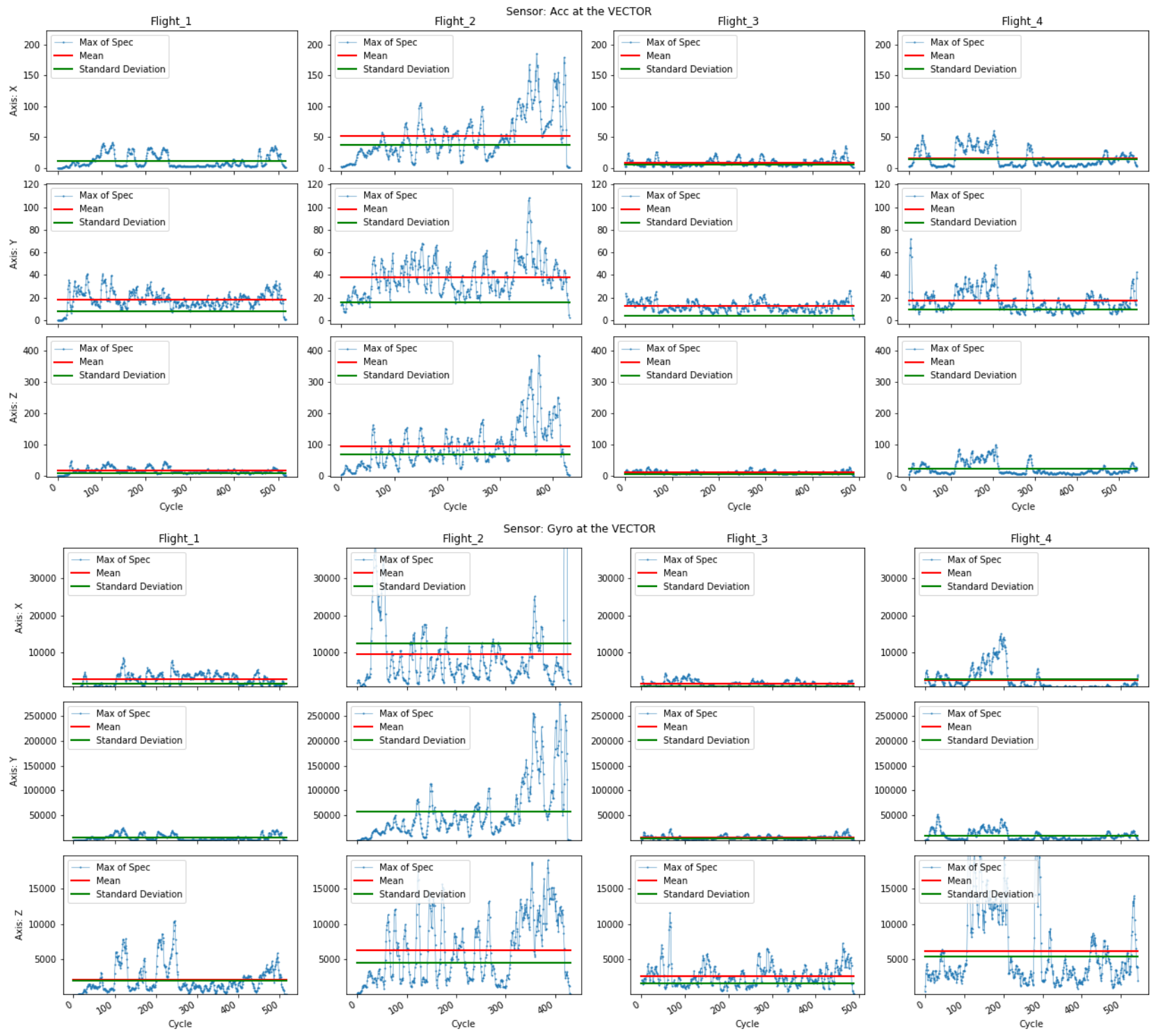

Figure 7. The progress of $\max (\mathrm{spec})$ from four different flights
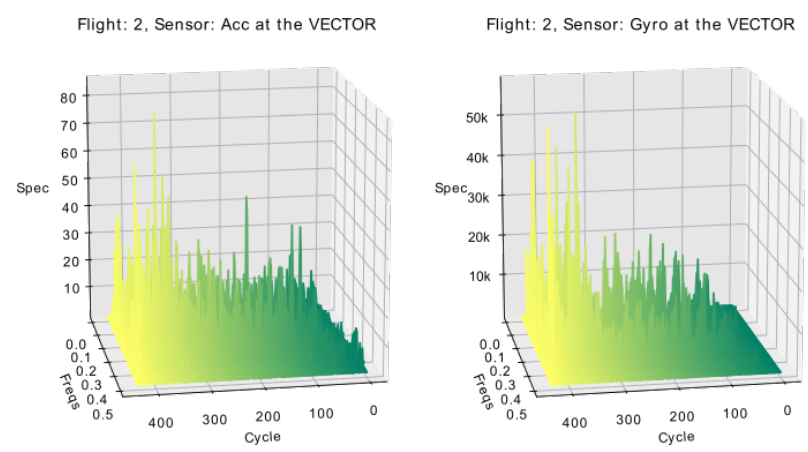

Figure 8. Periodograms of the Vector Sum

The maxima of each power spectrum estimation are illustrated in Figure 7 with an attempt to understand the fault
Table 2. Mean and standard deviation of max(spec) after the Vector Sum

$\begin{array}{llllll} & & \text { Flight_1 } & \text { Flight_2 } & \text { Flight_3 } & \text { Flight_4 } \\ \text { Acc } & \mu & 4.9 & \mathbf{1 4 . 2} & 4.1 & 6.7 \\ & \sigma & 2.1 & \mathbf{8 . 4} & 1.4 & 5.4 \\ & & & & & \\ \text { Gyro } & \mu & 969.3 & \mathbf{7 9 2 2 . 1} & 926.9 & 1527.7 \\ & \sigma & 769.6 & \mathbf{7 0 2 7 . 4} & 587.1 & 1397.21\end{array}$

progress. The results here and also in Table 1 indicate a relatively small difference in the mean of the accelerometer values, except for Flight 2. Since a higher mean or standard deviation score on this scale indicates a stronger anomaly in the operation, these descriptive statistics suggest that, as expected from the operational observation and extra payload, 

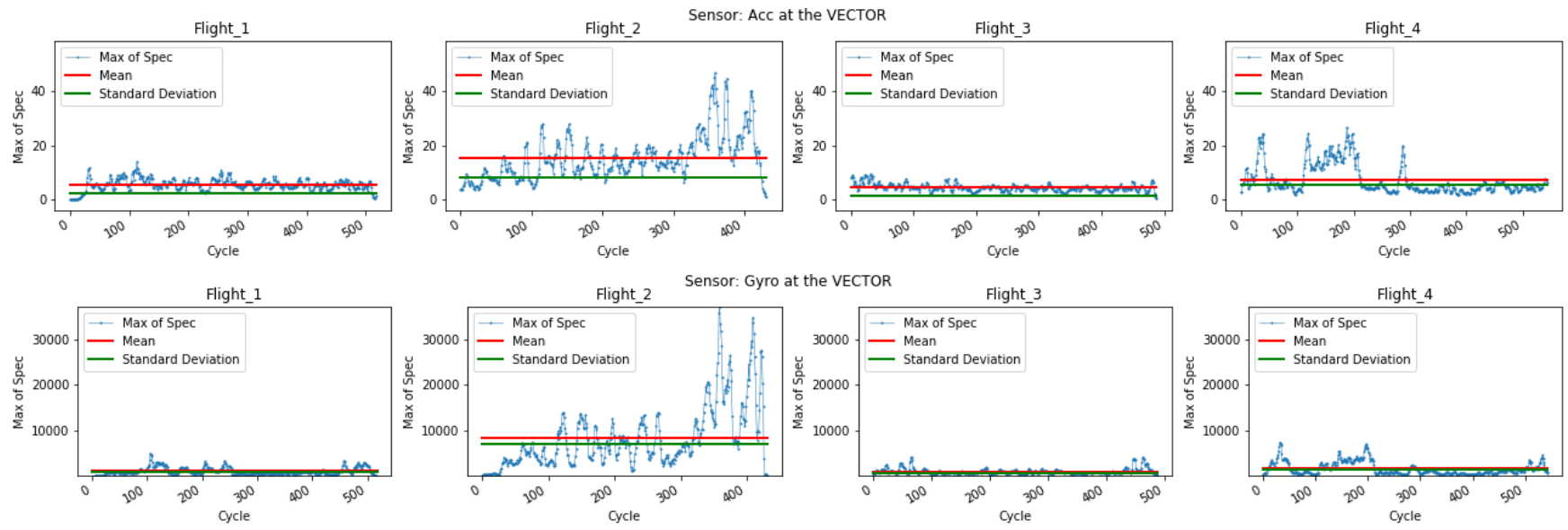

Figure 9. The progress of max (spec) of the vector sum from four different flights

second flight seem to have a considerably higher deterioration than the rest of the flights conducted by the same drone. The findings on the accelerometer may even hint a threshold level to indicate boundaries of the acceptance region of the vibration. Such a region can be regarded as a set of values of the vibration analysis for which the null hypothesis is not rejected. Depending on the acceptance region, if there is a significant number of critical values, the flight can be terminated and even an automated emergency landing can be performed as described in Bektash et al. (2020). However, the "Z" axis of gyro at the vector highlights that even a normal operation such like the one in Flight 4 may surpass the threshold value or alternatively the abnormal flight cannot be distinguished by the available information. This suggests that a particular vibration in a certain direction can lead or fail to demonstrate a failure, in spite of the fact that the other sensors perform differently. One way to overcome this issue to apply a "vector sum" to the three axes of a triaxial sensor to estimate the vibration overall magnitude which is independent of the axis and cannot be dominated by a single direction estimation.

The equation for a vector-sum (root-sum-of-squares) vibration magnitude consists of $\mathrm{X}, \mathrm{Y}$ and $\mathrm{Z}$ directions and is expressed as follows.

$$
\mathrm{VS}_{s}=\sqrt{X_{s}^{2}+Y_{s}^{2}+Z_{s}^{2}}
$$

So for the vibration levels above, the estimate of the spectral density of the vector sum would be as in Figure 8. Each power spectrum is calculated by the segmented windows of the square root of the sum of the squares of the directions. This correlates best with damage potential of all characterisations of the vibration movements. As the directions have different degrees of significance, some might have lesser or higher effect in causing damage. Accordingly, when considering a critical threshold value after which the flight can be terminated, Figure 8 gives clearly better results than individual directions. This is also valid for the maxima of each power spectrum (see Figure 9). In fact, the proposed Vector Sum method is able to indicate a comprehensible distinction between the normal and the high vibration flights. The framework can automatically separate between the regular state and the anomaly state without any required human intervention. Even though some false alarms can be found in different directions as like in the previous examples, Figure 9 provides a "scalar" quantity with only a single measurement. Logically, when the anomaly state occurs and a potential fault in the system is found, it is possible for the proposed model to perform a direct pattern recognition of measurements and detect a discrepancy between the readings and expected values. The higher mean and standard deviation scores of Flight 2 on Table 2 confirms this argument and can provide a coherent level of the acceptance region of the vibration.

\section{Conclusion}

This research contributes to UAS health monitoring literature by demonstrating the significance of vibration analysis in the context of estimating the power of a vibration signal at different frequencies. By using the Welch's method, the research tested the hypothesis that an in-flight anomaly can be detected by estimating the faulty status on the drones. The power spectrum estimations of segmented cycles are used as an indication of the failure. The results evidently reveal a notable correlation between condition monitoring data and over payload, indicating that a systematic process of data collection can be directly used for the condition evaluation of a drone's flight performance. Flight with normal operating conditions are used in the analysis as a way to ensure that the proposed method actually performs. Nevertheless, the research has not concluded the reason for the increasing degradation over operation of Flight 2 . This changeability might be explained by the various operational characteristics which are desirable for future work. 


\section{ACKNOWLEDGMENT}

We would like to thank Simon Jensen (Assistant Engineer, Department of Electronic Systems, Aalborg University) for his support and assistance in drone operations.

\section{REFERENCES}

Ali, J. B., Fnaiech, N., Saidi, L., Chebel-Morello, B., \& Fnaiech, F. (2015). Application of empirical mode decomposition and artificial neural network for automatic bearing fault diagnosis based on vibration signals. Applied Acoustics, 89, 16-27.

Ali, J. B., Saidi, L., Harrath, S., Bechhoefer, E., \& Benbouzid, M. (2018). Online automatic diagnosis of wind turbine bearings progressive degradations under real experimental conditions based on unsupervised machine learning. Applied Acoustics, 132, 167-181.

Amirat, Y., Benbouzid, M. E. H., Al-Ahmar, E., Bensaker, B., \& Turri, S. (2009). A brief status on condition monitoring and fault diagnosis in wind energy conversion systems. Renewable and sustainable energy reviews, 13(9), 26292636.

Balaban, E., \& Alonso, J. J. (2013). A modeling framework for prognostic decision making and its application to uav mission planning. In Annual conference of the prognostics and health management society (pp. 1-12).

Barbé, K., Pintelon, R., \& Schoukens, J. (2009). Welch method revisited: nonparametric power spectrum estimation via circular overlap. IEEE Transactions on signal processing, 58(2), 553-565.

Bechoefer, D. E. R., \& Capt, M. (2004). Rotor track and balance cost benefit analysis and impact on operational availability. In American helicopter society 60th annual forum, baltimore, $m d$.

Bektash, O., Pedersen, J. N., Ramirez Gomez, A., \& 1. CourHarbo, A. (2020). Automated emergency landing system for drones: Safeeye project. In 2020 international conference on unmanned aircraft systems (icuas) (p. 1056-1064). doi: 10.1109/ICUAS48674.2020.9214073

Bennett, R. M., \& Abel, I. (1982). Flight flutter test and data analysis techniques applied to a drone aircraft. Journal of Aircraft, 19(7), 589-595.

Chen, B., Matthews, P. C., \& Tavner, P. J. (2015). Automated on-line fault prognosis for wind turbine pitch systems using supervisory control and data acquisition. IET Renewable Power Generation, 9(5), 503-513.

Eleftheroglou, N., Zarouchas, D., Loutas, T., Mansouri, S. S., Georgoulas, G., Karvelis, P., ... Benedictus, R. (2019). Real time diagnostics and prognostics of uav lithiumpolymer batteries. In Proceedings of the annual conference of the phm society (Vol. 11).
EU. (2019a, March). Commission delegated regulation (eu) 2019/945 of 12 march 2019 on unmanned aircraft systems and on third-country operators of unmanned aircraft systems. Official Journal of the European Union.

EU. (2019b, May). Commission implementing regulation (eu) 2019/947 of 24 may 2019 on the rules and procedures for the operation of unmanned aircraft. Official Journal of the European Union.

Goebel, K., \& Saha, B. (2013). Prognostics applied to electric propulsion uav. Handbook of Unmanned Aerial Vehicles, 1053-1070.

Goebel, K., Smith, B., \& Bajwa, A. (2019). Ethics in prognostics and health management. International Journal of Prognostics and Health Management, 10(1).

Herrmann, R., Moortgat-Pick, A., \& Marx, S. (2019). Vibration analysis of structures using a drone (uav) based mobile sensing platform. In 5th international conference on smart monitoring, assessment and rehabilitation of civil structures.

Lai, J., Lv, P., Liu, J., \& Jiang, B. (2012). Noncommutativity error analysis of strapdown inertial navigation system under the vibration in uavs. International Journal of $A d$ vanced Robotic Systems, 9(4), 136.

Nguyen, P., Truong, H., Ravindranathan, M., Nguyen, A., Han, R., \& Vu, T. (2017). Matthan: Drone presence detection by identifying physical signatures in the drone's rf communication. In Proceedings of the 15th annual international conference on mobile systems, applications, and services (pp. 211-224).

Plasencia, G. N. M., Rodríguez, M. T., Rivera, S. C., \& Ángela Hernández López. (2012). Modelling and analysis of vibrations in a uav helicopter with a vision system. International Journal of Advanced Robotic Systems, 9(5), 220.

Proakis, J. G. (2001). Digital signal processing: principles algorithms and applications. Pearson Education India.

Saha, B., Koshimoto, E., Quach, C. C., Hogge, E. F., Strom, T. H., Hill, B. L., ... Goebel, K. (2011). Battery health management system for electric uavs. In 2011 aerospace conference (pp. 1-9).

Saha, B., Quach, C. C., \& Goebel, K. (2012). Optimizing battery life for electric uavs using a bayesian framework. In 2012 ieee aerospace conference (pp. 1-7).

Saidi, L., Ali, J. B., Bechhoefer, E., \& Benbouzid, M. (2017). Wind turbine high-speed shaft bearings health prognosis through a spectral kurtosis-derived indices and svr. Applied Acoustics, 120, 1-8.

Schuster, A. (1898). On the investigation of hidden periodicities with application to a supposed 26 day period of meteorological phenomena. Terrestrial Magnetism, 3(1), $13-41$. 
Simsiriwong, J., \& Sullivan, R. W. (2012). Experimental vibration analysis of a composite uav wing. Mechanics of Advanced Materials and Structures, 19(1-3), 196-206.

Smith III, J. O. (2011). Spectral audio signal processing. W3K publishing.

UAS-ability. (n.d.). Home, [youtube channel]. Retrieved 2020-08-25, from https://www.youtube . com/channel / UCwIUbrNZCwBuWZ4 rRBUq3 LA

Verbeke, J., \& Debruyne, S. (2016). Vibration analysis of a uav multirotor frame. In Proceedings of isma $2016 \mathrm{in-}$ ternational conference on noise and vibration engineering (pp. 2401-2409).
Welch, P. (1967). The use of fast fourier transform for the estimation of power spectra: a method based on time averaging over short, modified periodograms. IEEE Transactions on audio and electroacoustics, 15(2), 70-73.

Yang, W., Tavner, P., \& Wilkinson, M. (2009). Condition monitoring and fault diagnosis of a wind turbine synchronous generator drive train. IET Renewable Power Generation, 3(1), 1-11.

O. Bektash Web of Science ResearcherID: ABB-7971-2020 publons.com/researcher/3373343 